\title{
La relación entre público y privado en los Servicios Sociales: el papel del Tercer Sector
}

\author{
The Public-Private Relationship in Social Services: \\ The Role of the Third Sector \\ Àngels Guiteras Mestres \\ Presidenta de la Taula del Tercer Sector Social en Cataluña \\ presidenci@tercersector.cat
}

Recibido: $10 / 11 / 2011$

Revisado: 24/11/2011

Aceptado: 12/12/2011

Disponible on line: 15/02/2012

\section{Resumen}

La alianza estratégica entre la Administración Pública y el Tercer Sector es clave para el desarrollo del modelo de gestión de los Servicios Sociales en general, en el futuro, y muy concretamente para la gestión externalizada de servicios en los que tiene un papel protagonista, dada la solvencia social del Tercer Sector y su capacidad de multiplicar los fondos públicos. El Tercer Sector es un actor emergente y aporta eficiencia y cohesión social. Debemos avanzar en medir su impacto y su valor añadido específico, en la identificación de cláusulas sociales que se utilicen en la contratación pública, en el fortalecimiento y la vertebración del sector, así como en la capitalización y profesionalización de las entidades sociales. Se identifican algunos de los principales desafíos de la relación público-privado y algunas propuestas de futuro.

Palabras clave: Tercer Sector, publico, eficiencia, cláusulas sociales, cohesión social

\begin{abstract}
The strategic alliance between public administration and the Third Sector is key for the development of a management model for social services in general in the future, and specifically for the externalized management of services in which it acts as a protagonist, given the social solvency of the Third Sector and its ability to multiply public funds. The Third Sector is an emerging player and contributes efficiency and social cohesion. We should move forward in measuring its impact and specific value added by identifying the social clauses which are used in public procurement, in the strengthening and structuring of the sector as well as in the capitalization and professionalization of social entities. Some of the principal challenges of the public-private relationship are identified and some proposals for the future are put forth. Keywords: Third Sector, public, efficiency, social clauses, social cohesion.
\end{abstract}

Referencia normalizada: Guiteras Mestres, A. (2012): «La relación entre público y privado en los Servicios Sociales: el papel del Tercer Sector». Cuadernos de Trabajo Social, 25(1): 125-132.

Sumario: 1. ¿Qué es el Tercer Sector? 2. ¿Qué entendemos por relación público-privado. 3. La importancia del modelo de relación público-privado en el avance de los Servicios Sociales. 4. La situación actual. 5. La situación actual. 6. Los principales retos. 7. Algunas propuestas. 8. Referencias bibliográficas.

\section{1. ¿Qué es el Tercer Sector?}

La marca Tercer Sector como tal es muy reciente en España y es fruto del trabajo conjunto que han ido realizando diversas entidades y organizaciones que, compartiendo objetivos comunes de transformación social hacia una sociedad más justa, con menos desigualdades y más cohesión social, representan el espacio más o menos organizado, según el territorio del llamado Tercer Sector.

En Europa tiene diferentes denominaciones. En Francia se denomina Tiers-secteur, aunque es un concepto que se utiliza poco, ya que suelen utilizar el término de «economía social». En 
Alemania, por su parte, se habla de entidades «de interés general»; en Italia y en el Reino Unido es donde más se utiliza esta expresión: Terzo Settore y Third Sector, respectivamente. En Estados Unidos y en Canadá se conoce como sector Non Profit. Variedad de nomenclaturas que también nos encontramos en nuestras entidades: entidades sin afán de lucro, entidades no-lucrativas, entidades de iniciativa social, organizaciones no gubernamentales (ONG, oenegés sociales u oenegés del cuarto mundo). Una variedad de denominaciones todas ellas validas, pero como sector necesitamos visualizarnos con una denominación común. Hoy por hoy, el término Tercer Sector es una marca con potencial de futuro, aunque no cumple algunos de los criterios básicos de comunicación: que el nombre por si solo ya defina y comunique de qué se trata y dé información suficiente. Actualmente hay que explicar qué se entiende por Tercer Sector. Todos sabemos, cuando se trata de la Administración, a quién nos estamos refiriendo, aunque no todos saben que se trata del primer sector; también todos sabemos que las empresas mercantiles constituyen lo que se conoce como el segundo sector. En nuestro caso se produce el fenómeno a la inversa. Se conocen muchas entidades, tanto de voluntariado como de servicios, que llevan a cabo un sinfín de actividades desde la proximidad en diferentes ámbitos; infancia, personas mayores, mujeres, inmigración, drogodependencias, personas con discapacidad, pero no se las relaciona directamente con el nombre Tercer Sector. A pesar de ello, esa debilidad es también su fuerza. Un marchamo en el que caben muchas concepciones y puede estar representada toda la riqueza y diversidad del sector, una marca global, una marca para todos, una marca con potencial de futuro. Una debilidad que puede representar su oportunidad.

El Tercer Sector social en España es una realidad muy compleja, plural y diversa. Como otros sectores, tiene gran relevancia el que podamos conocer con la máxima exactitud posible su dimensión económica y social. No hemos tenido datos hasta que se publicó el primer anuario español, titulado Anuario del Tercer Sector de Acción Social en España en 2011.

Los datos globales señalan que el Tercer Sector social en España está representado por 29.000 entidades u organizaciones, de las cuales el 92,8 por ciento son entidades del llamado primer nivel ${ }^{1}$, el 4,7 por ciento son de segundo nivel $^{2}$ y el 2,5 por ciento lo integran mayoritariamente entidades de tercer nivel. ${ }^{3} \mathrm{La}$ mayoría de estas entidades son en su forma jurídica asociaciones: en concreto el 69,5 por ciento y fundaciones el 11 por ciento. El número de personas voluntarias se estima en 873.171 y el de profesionales es 530.000 , que constituye el 2,7 por ciento del mercado general de trabajo. El Tercer Sector social representa entre el 1,42 por ciento y el 1,62 por ciento del PIB español (2008). Las actividades de las entidades del Tercer Sector de acción social están dirigidas principalmente a cuatro grupos: personas con discapacidad, infancia o menores de ambos sexos, personas mayores y población general. El número de beneficiarias asciende a 42.000.000 personas.

Disponer de estos datos nos ayuda a conocer la representación del sector y a poner en valor su aportación como agente social en la realidad económica y productiva de nuestro país. El reconocimiento del Tercer Sector social como un agente social de primera división requiere voluntad política, pero también de que dicho sector esté rigurosamente organizado y estructurado, atendiendo a las diversas realidades territoriales e impulsando la estructuración del mismo en las comunidades autonómicas. Una buena vertebración y organización es imprescindible para un actor emergente como es el Tercer Sector.

Desde mi punto de vista, esta fuerza y este potencial es el reto de los territorios: estructurar una representación a nivel nacional que tenga en cuenta la realidad y cómo se ha ido realizando hasta hoy y cuáles son las principales organizaciones que se han configurado. No es suficiente con que las entidades trabajen a nivel de los territorios, de lo que se trata es de que se unan territorialmente y que juntas pue-

\footnotetext{
${ }^{1}$ Entidades de primer nivel: son aquellas entidades de base que se representan a sí mismas.

2 Segundo nivel: son entidades como federaciones o fundaciones que funcionan como entidades paraguas y están formadas por entidades de primer nivel, en general de un mismo ámbito de actuación.

${ }^{3}$ Tercer nivel: son plataformas formadas por las entidades llamadas de segundo nivel.
} 


\begin{tabular}{|l|l|l|}
\hline & \multicolumn{1}{|c|}{$\mathbf{2 0 0 3}$} & \multicolumn{1}{c|}{$\mathbf{2 0 0 9}$} \\
\hline Número de entidades & Más de 5.600 & En torno a 7.500 \\
Volumen económico catalán & Casi un $1 \%$ del PIB catalán & Un 2,8\% del PIB \\
Número de personas contratadas & Más de 52.000 & Más de 100.000 \\
Número de personas voluntarias & Más de 155.000 & Más de 245.000 \\
Número de personas destinatarias & Más de 1.000 .000 & Más de 1.700 .000 \\
\hline
\end{tabular}

Tabla 1. Evolución de los grandes números (2003-2009).

dan tener un mayor nivel de representación y de interlocución, que les permita avanzar en los cambios que se necesitan para conseguir al máximo los objetivos sociales que como sociedad nos planteamos. Trabajar en consenso desde la pluralidad del sector nos fortalece, tanto a las distintas entidades como a la sociedad, y nos hace singulares. Un exceso de protagonismo de algunos y la idea de que es suficiente entender la territorialidad como la multipresencia de una entidad en varios territorios, puede enlentecer la formación de una organización fuerte y eficiente a nivel del Tercer Sector social en España.

Una cosa es estar en el territorio y otra muy distinta es estar organizado como sector territorialmente. En este sentido, Cataluña ha sido pionera: en el año 2003 constituyó y organizó la Taula de Entidades del Tercer Sector Social de Catalunya, que supone la estructuración, por primera vez en España, del Tercer Sector social y se convierte en una referencia de otras comunidades autonómicas. La Taula del Tercer Sector Social es una organización de tercer nivel, agrupa a treinta federaciones o agrupaciones de entidades que aglutinan a más de 4.000 entidades no lucrativas, que actúan en el campo social: asociaciones, cooperativas, fundaciones, empresas de inserción y centros especiales de trabajo.

La Taula del Tercer Sector tiene como objetivo velar por la existencia de más y mejores políticas sociales y por la consolidación y el reconocimiento del Tercer Sector. Se propone una doble misión: por un lado, la de incidir en las políticas sociales catalanas con el objetivo de mejorar el bienestar de las personas y conseguir su inclusión social y, por otro, vertebrar y fortalecer el propio sector, consolidar las entidades y obtener el reconocimiento de su acción social por parte de los sectores económi- cos, las administraciones públicas y el conjunto de la sociedad catalana.

La visión que inspira esta misión es el deseo de conseguir que las organizaciones del Tercer Sector social sean motor de transformación social y creación de futuro colectivo, trabajando para lograr una sociedad en la que los derechos sociales de todas las personas sean reales y efectivos y un país en dónde todas las personas participen activamente en la creación de su futuro y un espacio público de participación ciudadana en dónde la aplicación y ampliación de los derechos civiles para todos consiga retos de alta cohesión social.

El Tercer Sector social en Cataluña avanza unido y ha podido constatar su crecimiento desde los datos que se publicaron en el Libro blanco de acción cívica del año 2003 hasta la realidad de la representación del sector publicada en el Anuario del año 2009, cuyos datos publicados en el Anuario Tercer Sector Social de Cataluña 2009, figuran en la tabla 1.

\section{La relación entre público y privado}

¿Qué entendemos por público? Lo público va más allá de la Administración, aunque ésta representa y regula lo público. De hecho deberíamos hablar de Administración pública y sector privado, ya sea mercantil o no lucrativo. Lo público somos todos y todas. Lo privado también es público, tiene una dimensión social y pública que no se puede olvidar. El Tercer Sector es privado, pero tiene una incidencia pública más importante que lo mercantil, reinvierte los beneficios en el bien común y articula la fuerza del voluntariado. Es la parte privada que tiene más papel en lo público, en la construcción de lo de todos y todas.

El Tercer Sector incorpora el voluntariado y el compromiso cívico, es una manera de que las personas participen en el compromiso cívi- 
co. La Administración, los políticos son los que representan a toda la ciudadanía y han de representar los intereses de todos. Todos y todas formamos parte de lo que es la construcción social e incidimos en lo público.

\section{La importancia del modelo de relación público y privado en el avance de los Servi- cios Sociales}

Durante los últimos años, la Administración pública ha ido construyendo el Estado del bienestar y se han puesto en funcionamiento diversos servicios de atención social a las personas. Esta dinámica de crecimiento y puesta en marcha de recursos por parte de la Administración se ha realizado con un espíritu empresarial, en el sentido de que ha realizado funciones de gestión parecidas a los de las empresas mercantiles, buscando la máxima eficiencia. Se ha potenciado el desarrollo de los servicios desde la propia Administración, que ha gestionado internamente y ha creado en ocasiones consorcios que se han encontrado con las mismas dificultades en la gestión que se han detectado en la Administración: falta de flexibilidad y adaptabilidad de las jornadas con los horarios que serían necesarios para la prestación de servicios de calidad, adaptados a las necesidades reales de las personas beneficiarias, incremento de costes, etc. En las ocasiones en que se ha planteado la externalización del servicio, se ha llevado a cabo más por dificultades presupuestarias internas que no permitían gestionar de manera directa, que por una voluntad real de externalizar el servicio. Por ello en general la externalización no responde a un modelo claro y tiene dificultades tanto para la Administración que contrata, como para la entidad o empresa contratada y lo que es más importante, para la sociedad en general. En la práctica, la Administración no está sólo proporcionando un servicio, sino que también quiere producirlo. En realidad en muchos casos pretende utilizar al contratante como mero suministrador de personal, a la vez que destina funcionarios para que realicen también las mismas actividades, creando una cierta confusión entre control y evaluación y la ejecución del servicio.

Mediante un ejemplo como el de la externalización de los servicios de atención domiciliaria se puede comprender mejor el fenómeno. La atención social domiciliaria, conocida co- mo SAD, se externaliza. Se trata de servicios complejos y de gestión intensiva de personal. Con dicha medida se pretende profesionalizar el servicio, aportar mayor calidad en la intervención y mayor eficiencia. Si la externalización no es la adecuada pueden darse situaciones en las que se producen intervenciones en paralelo por parte de los profesionales de la empresa contratada y de los funcionarios que no han cambiado su rol, a quienes se les indica que actúen como si fueran quienes estuvieran a cargo de la producción del servicio. En uno de los procedimientos de inicio de un caso de atención en el domicilio, el protocolo de la presentación al usuario en el domicilio se produce con un trabajador social de la administración, un trabajador social de la empresa que tiene el encargo y un trabajador familiar de la empresa que es quién acudirá normalmente al domicilio. Nos encontramos en un mismo acto con la persona usuaria, beneficiaria del servicio y con tres profesionales, dos de los cuales trabajadores sociales. Unos controlan a los otros, se duplican funciones y se consigue el resultado contrario al que se pretendía y, con ello, la máxima ineficiencia.

Podemos comprender mejor lo que ocurre en muchas de las externalizaciones actuales, con otro ejemplo: es como si cuando fuéramos al médico de nuestro centro de Atención Primaria (CAP) o al ambulatorio, nos atendieran dos médico: uno funcionario y el otro de la empresa gestora del mismo. Lo podemos aplicar también a los hospitales, ya que tienen externalizada la gestión.

El modelo de relación entre público y privado debe redefinir el papel de cada uno de los actores y tender hacia un nuevo modelo basado en lo que se conoce como gobernanza democrática.

En gobernanza democrática el gobierno ya no imita a la empresa mercantil, sino que los valores de lo público, garantizados por la responsabilidad pública del gobierno en la programación, evaluación y gestión, son los que guían la producción o gestión externalizada de servicios financiada con fondos públicos». (Pascual, 2011).

El Tercer Sector forma parte de la denominada esfera pública compartida y una parte de esa relación se concreta en la prestación de ser- 
vicios a través de subvenciones, convenios, o contratos. Deberíamos tender hacia una relación más estable. Las subvenciones son importantes para el desarrollo de muchas iniciativas y la puesta en marcha de programas de innovación social, pero también son precarias: en pocas ocasiones tienen carácter de plurianualidad y dificultan la planificación y la ejecución de los programas y servicios que se deben llevar a cabo. A este respecto, se han propuesto una serie de medidas para mejorar la Ley General de Subvenciones. La prestación de servicios también se lleva a cabo a través de contratos regulados por la Ley de Contratos del Sector Público. Esta ley regula, por primera vez, el Tercer Sector y la contratación pública y pone en común unas mismas reglas de juego para el sector mercantil y el sector no lucrativo, que en ocasiones no sólo no tienen en cuenta la especificidad del sector sino que lo discriminan, como es el caso de la concurrencia simultánea Para un mismo objeto de contrato, entidades no lucrativas y entidades mercantiles deben concursar por el importe neto y no por el importe del presupuesto de licitación, con IVA incluido. Se valoran las ofertas sin incluir el coste del IVA. Este hecho lleva a una situación paradógica, que crea dos situaciones en la práctica: se paga por un servicio adjudicado el valor de la oferta en el caso de una entidad del Tercer Sector y, si se trata de una entidad privada mercantil, a la adjudicataria del servicio, se le paga el valor de la oferta más el importe del IVA que puede ser un 7 por ciento o un 18 por ciento más, según la actividad de la que se trate.

En el caso de las entidades del Tercer Sector, al estar exentas por imposición legal del impuesto del IVA, dicha exención impide recuperar el IVA soportado y deja en peor situación para la ejecución de nuestras actividades por las cuales se paga por lo mismo que exige el contrato a todos, menos dinero. Este es uno de los ejemplos y una de las cuestiones prácticas que debe resolverse de la relación de la Administración con el Tercer Sector.

Las entidades y organizaciones tienen, que buscar, como otras fórmulas de financiación para adelantar el dinero de las subvenciones y para gestionar los retrasos en el pago por parte de las Administraciones. Financiar y ver cómo pueden, a través de sus fondos propios, de otras vías de ingreso o de créditos, hacer viable la realización de los proyectos. Creo que, aún hoy, debemos explicar que cuando se habla de las subvenciones al sector, no son ayudas a fondo perdido ni gratuitas, ni tampoco financiación a las entidades para que asegurar su simple existencia.

Las entidades sociales no viven de las subvenciones públicas. Estas subvenciones, al contrario de lo que ocurre en otros sectores, siempre han sido precarias y han generado mucha inseguridad a las entidades, a sus profesionales y a las personas beneficiarias.

El Tercer Sector se sostiene, fundamentalmente, con la fuerza del voluntariado. Las subvenciones que recibe, al igual que ocurre con los contratos, son, en la mayoría de los casos, en concepto de contraprestación de servicio. Hemos de avanzar en la relación entre público y privado para mejorar la eficiencia de nuestras actuaciones, siendo conocedores de que con nuestras acciones se consigue un mayor impacto de los fondos públicos que son de todos.

En la relación entre público y privado es importante trabajar conjuntamente en la identificación e inclusión de cláusulas sociales en la contratación pública. En este caso lo que nos encontramos es que el marco legal lo hace posible, la voluntad política existe pero las acciones y la metodología hacen que en la práctica estas cláusulas sean débiles y tengan escasa presencia en los pliegos de las convocatorias de contratación pública. En ocasiones quedan reducidas a la contratación reservada, a cláusulas medio-ambientales y a algunas cláusulas de contratación laboral para colectivos con dificultades especiales.

Las cláusulas sociales son, como su nombre indica, aspectos sociales, que se pueden incluir en las bases de contratación pública y pueden ser valorados y por tanto mesurables. Pueden plantearse tanto como criterios de admisión de solvencia social en general y también en los denominados contratos de reserva, como en los criterios de valoración y puntuación en la fase del proceso de adjudicación y como una obligación inherente del contrato. La implementación de las cláusulas sociales es de gran importancia estratégica y de gran valor en términos de eficiencia social y tendrá un papel clave en el desarrollo de la cartera de servicios sociales 
y de los servicios de promoción de la autonomía de las personas y de atención a la dependencia en los próximos años.

Para medir el valor añadido específico del Tercer Sector en la prestación de servicios públicos y consolidar el papel que las entidades no lucrativas han desarrollado hasta hoy en la gestión y la provisión de estos servicios, debe avanzarse en la identificación de indicadores específicos, de sector, de entidades y de proyectos.

\section{El sistema de indicadores de valores del Tercer Sector social}

\subsection{Indicadores globales de sector}

Se trata de aquellos indicadores que pretenden poner en relieve los hechos diferenciales del Tercer Sector Social y el impacto de su actividad. Por ejemplo: el volumen de ocupación del Tercer Sector social.

\subsection{Indicadores de entidad}

Se trata de aquellos indicadores que pretenden poner en relieve los hechos diferenciales de cada entidad del Tercer Sector Social y el valor añadido de su actividad. Por ejemplo: Participación del usuario en la definición y la mejora de servicios (número de horas de reuniones).

\subsection{Indicadores de proyecto}

Se trata de aquellos indicadores que pretenden poner en relieve la aportación diferencial de cada entidad en el proyecto objeto del concurso. Por ejemplo: Dimensión comunitaria del proyecto.

\subsection{Tipo de Indicadores}

\subsubsection{Sector}

1.1. Volumen presupuestario del Tercer Sector social

1.2. Peso de la financiación privada en el presupuesto del Tercer Sector social

1.3. Volumen económico aportado por la base social

1.4. Volumen de usuarios del Tercer Sector social

1.5. Volumen económico de la aportación de la base social por usuario

1.6. Peso del Tercer Sector social en la economía catalana
1.7. Volumen de ocupación del Tercer Sector social

1.8. Volumen de trabajo voluntario del Tercer Sector social

1.9. Peso de los voluntarios en la población catalana

1.10. Peso de la base social del Tercer Sector social

\subsubsection{Entidad}

2.1. Ratio de usuarios/beneficiarios en situación de exclusión social

2.2. Volumen de personas sensibilizadas con valores y derechos sociales

2.3. Volumen de servicios propios y accesibles

2.4. Voluntariado en la entidad

2.5. Tasa de contratación indefinida

2.6. Volumen de la base social de la organización

2.7. Participación y trabajo en red

2.8. La primacía de las personas y de la misión por encima del lucro en la política de la organización

2.9. Incidencia de las actuaciones innovadoras

2.10. Transferencia de conocimiento

2.11. Retorno social

\subsubsection{Proyecto}

3.1. Dimensión comunitaria del proyecto

3.2. Volumen de personas en riesgo o en situación de exclusión social contratadas para la ejecución del proyecto

3.3. Participación del usuario en el diseño y la mejora del servicio

3.4. Complementariedad de servicios

3.5. Voluntariado en el proyecto

3.6. Información y sensibilización social sobre la materia objeto del proyecto

3.7. Participación democrática en el proyecto

3.8. Participación con la Administración en la identificación de nuevas necesidades

3.9. Volumen de acuerdos para el trabajo en red

3.10. Conocimiento del territorio local

3.11. Adaptación a la identidad sociocultural local

3.12. Transferencia de conocimiento en el proyecto

3.13. Retorno social local 


\section{La situación actual}

Estamos en plena recesión económica y las políticas de reducción del déficit público están a la orden de día. En esta situación, las necesidades sociales cambian y aumentan día a día. Las políticas sociales son prioritarias y están vinculadas a las políticas de crecimiento económico. $\mathrm{La}$ inversión en política social debe ser una apuesta clara. La relación de la Administración Pública con el Tercer Sector es estratégica y se irá fortaleciendo en los próximos años. El Tercer Sector es un actor emergente que aporta capital social. Su impacto debe potenciarse y debemos encontrar fórmulas de colaboración que superen las dificultades actuales en la relación con la Administración pública. Respondiendo a esa nueva manera de hacer, el papel de la sociedad civil, la participación ciudadana y las entidades del Tercer Sector tienen un protagonismo, como responsables de lo público, tanto en los temas de solidaridad y voluntariado como en los de prestación de servicios que cada día más son producidos por entidades del Tercer Sector.

No existe dicotomía entre entidades de voluntariado o entidades prestadoras de servicios, con o sin la colaboración de voluntarios. No existe tal esquizofrenia y definir la complejidad del Tercer Sector social implica reconocerlo en la diversidad y las distintas organizaciones. El Tercer Sector es más amplio que el concepto de economía social. Una parte del Tercer Sector que realiza actividad económica es también economía social. Algunas posturas interesadas plantean el debate para debilitar el potencial del Tercer Sector social en la prestación de servicios de atención a las personas y poder apartarlo de su campo de competencia y de la economía productiva ,en dónde lo visualizan como un fuerte competidor por su aportación de capital social, valores y eficiencia social.

\section{Los principales retos}

Principalmente son: 1) el proceso de desarrollo e implementación de las Leyes de Servicios Sociales en las comunidades autónomas, Leyes de derechos y oportunidades de la infancia y la adolescencia, Leyes de la vivienda y de la Ley de la promoción de la autonomía personal y la dependencia. 2) la definición de amplias carteras de servicios que tienen que dar cobertura a prestaciones en diferentes ámbitos.

En un momento en que cada día hay más necesidades y menos recursos para cubrirlas, el
Tercer Sector tiene una función primordial por su naturaleza: aportar más valor, multiplicando los recursos y aportando capital social tanto por la reinversión de los beneficios en la misión como en el valor añadido del voluntariado, de los profesionales que llevan a cabo los programas implicados en la misión de la entidad.

El importante avance que pueden suponer estas leyes en la profundización del Estado del bienestar y la modernización de las políticas sociales en nuestro país así como el marco legislativo del que nos hemos dotado nos permite y obliga a poner en marcha nuevas prestaciones, nuevos servicios. La situación actual es una oportunidad y una necesidad de repensar un nuevo modelo de crecimiento e implementación de los Servicios Sociales para que estos den la máxima cobertura y permitan el desarrollo y la autonomía de la sociedad que nos plantea nuevos retos de conciliación familiar y laboral, de gestión de la diversidad, etc.

El desarrollo de los Servicios Sociales debe ser garantizado por la Administración, pero diseñados conjuntamente con la participación de la sociedad y muy en particular con la implicación de las entidades sociales que tienen vida propia en los territorios, cerca de las diferentes realidades y trabajando en red con los diferentes agentes sociales de un mismo lugar. Unos Servicios Sociales en los que todos estemos implicados, seamos corresponsables y aportemos aquello a lo que nos comprometamos. Unos servicios sociales que no nos vengan dados.

Las leyes de Servicios Sociales nos aportan derechos subjetivos, universales, que han de garantizarse desde la Administración, con el protagonismo de las entidades del Tercer Sector .

El Anuario 2011 del Tercer Sector Social de Catalunya, realizado conjuntamente por la Taula de Entidades del Tercer Sector social y el Observatorio del Tercer Sector, distinguen y analizan cuatro tipos de retos: retos en un entorno de crisis, retos en la gestión y el desarrollo de las personas, retos organizativos y retos en el ámbito relacional. Señalaré algunos de los principales, para que este sector pueda ejercer un papel clave en la relación con la Administración pública.

1. El fortalecimiento de las estructuras económicas y financieras de las entidades del Tercer Sector. 
2. Apostar por incorporar criterios de gestión para mejorar la eficacia y la eficiencia de las entidades.

3. Medir el impacto y evidenciar el valor añadido específico del sector.

4. Impulsar la cultura de la transparencia y la rendición de cuentas.

5. La formación, en especial de los directivos, en gestión de recursos humanos, económicos y financieros.

6. Mantener la identidad de los servicios sociales.

7. Desarrollar un modelo eficiente propio que no se fagocite en el ámbito socio-sanitario.

8. Desarrollar servicios socio-sanitarios propios dentro del modelo social.

La evaluación del valor añadido específico que aporta el Tercer Sector es uno de los principales retos de las entidades y una de las responsabilidades públicas de la gestión externalizada de los servicios que se financian con fondos públicos. El impacto de nuestras acciones, la eficiencia social, no la podemos dar por supuesta. Medir la eficiencia social del Tercer Sector fortalecerá su reconocimiento en todos los ámbitos, también en el de la contratación pública. Avanzar en esta línea es también avanzar en otro de los retos: la identificación de cláusulas sociales y su utilización en las condiciones y requerimientos contractuales en el ámbito de los servicios de atención a las personas en un sentido amplio. Se tienen que identificar unos indicadores claros, válidos, confiables y comparables que midan los resultados.

\section{Algunas propuestas}

El Anuario 2011 del Tercer Sector Social de Cataluña propone cinco temas clave para la mejora del Tercer Sector social:

1. Desarrollo de las herramientas y capacidades de la gestión. Para conseguir que la mayoría de las entidades funcionen con eficacia y eficiencia en el cumplimiento de sus misiones y en la realización de sus actividades.

2. Creación de capacidad de inversión del Tercer Sector Social. Esta capacidad le posicionara mejor como operador destacado de servicios sociales públicos.

3. Impulso a la colaboración y la integración para ganar dimensión en la actuación. Poner en valor la capilaridad del sector y su capacidad de colaborar entre si. Un Tercer Sector Social vertebrado y estructurado con entidades de segundo y tercer nivel que son un espacio activo de colaboración y encuentro entre las entidades de base, colaborando en la mejora del funcionamiento.

4. Crecimiento de la base social, de su implicación y del compromiso económico. Una base social amplia y comprometida con la misión, tendrá capacidad de acción e independencia económica para sus actuaciones.

Este proceso de reconocimiento como actor social relevante del Tercer Sector y como actor social de primer orden, le hará tener un papel activo y fundamental en la gobernanza de nuestro país y una relación positiva con la Administración pública, basada en la confianza mutua.

\section{Referencias bibliográficas}

Equipo de Investigación Sociológica, S.A (EDIS) Fundación Luis Vives (coord.) (2010). Anuario del Tercer Sector de Acción Social en España. [s.n]. Madrid

Pascual Esteve, J.M (2008). El Tercer Sector un actor emergente en gobernanza democrática. El papel de la ciudadanía en el auge y decadencia de las ciudades. El fin del gerencialismo o la recuperación de lo público y sus actores. Valencia: Ed. Tirant lo Blanch

Taula d'entitats del Tercer Sector Social de Catalunya y Observatori del Tercer Sector. (2009). Anuario 2009 del Tercer Sector Social de Catalunya. [s.n].

Taula d'entitats del Tercer Sector Social de Catalunya (2009). El Valor añadido del Tercer Sector en la prestación de servicios públicos. Guía práctica de cláusulas sociales en la contratación pública de servicios sociales y de atención a las personas [s.n]. Barcelona

Taula d'entitats del Tercer Sector Social de Catalunya y Observatorio del Tercer Sector. (2011). Anuario 2011 del Tercer Sector Social de Catalunya [s.n]. Barcelona. 